\title{
La arquitectura popular en la Edad Media (Guipúzcoa)
}

\author{
Beatriz Arizaga Bolumburu *
}

Ante este tema nos encontramos con una ausencia total de estudios bibliográficos referentes a la vivienda popular urbana. En contraposición, los estudios sobre la casa popular rural están más generalizados. La mayor parte de estos estudios se realizaron en la primera mitad de este siglo, destacando por su calidad los trabajos de Baeschlin sobre «La arquitectura del caserío vasco", y los de los arquitectos Guimon y Muguruza sobre "el caserío vasco» y «la casa rural en el país vasco» respectivamente. Ninguno de los trabajos citados toca el tema de la vivienda popular urbana, y tenemos que esperar a los trabajos de Yrizar sobre "las casas vascas", para ver aparecer tímidamente las viviendas urbanas reflejadas en dicho estudio. Quien con posterioridad to ha tratado en diversos artículos ha sido el historiador y antropólogo Julio Caro Baroja, refiriéndose especialmente a Navarra ${ }^{1}$.

* Universidad de Cantabria.

A. Baeschlin: La Arquitectura del caserío vasco, Bilbao 1980. Primera Edición Barcelona 1930.

GuImón: "El caserío Basco", Artículo publicado en Revista de Arquitectura, n. ${ }^{\circ} 1713$ año 1919 , págs. $120-124$.

Muguruza: "La casa rural en el País Vasco", Artículo publicado en Revista de Arquitectura, $\mathrm{.}^{\circ} 17$ año 1919 , págs. 244-248.

J. Yryzar: Las casas vascas, Edit. Biblioteca Vascongada Villar. Bilbaro 1980. Primera Edición 1930.

J. Caro Baroja: Los Vascos, Edit. Istmo. Madrid 1971.

-: "Algunas notas sobre la casa en la villa de Lesaka», Artículo publicado en EuskoFolklore 1929, t. IX, págs. 69-91.

-: La casa en Navarra. Pamplona 1983. 
A pesar de esta realidad trataré de ofrecer ciertos datos referentes a la vivienda urbana medieval en el Norte Peninsular, concretamente, en Guipúzcoa. Para este trabajo he contado con la documentación escrita de la época, con vestigios arquitectónicos que han pervivido y con ciertos documentos gráficos del período bajomedieval. Todo ello ha permitido hacernos una idea bastante exacta de cómo fue la vivienda popular urbana en la Edad Media.

Planteamos aquí el tema de la arquitectura popular urbana por un motivo que consideramos fundamental, que es la implantación y desarrollo del sistema urbano en todo el territorio guipuzcoano durante los siglos XIII y xIV. En Guipúzcoa se fundan y establecen veinticinco núcleos de población, la creación de tal elevado número de núcleos urbanos en un espacio tan limitado, va a suponer la introducción en Guipúzcoa de los rasgos característicos del mundo urbano medieval europeo. Los espacios intramuros están privilegiados, sus vecinos adquieren un estatuto sociopolítico específico, que mejorará sus anteriores condiciones, se emprenderá la diversificación social del trabajo y comenzarán a gobernarse con autonomía entre ellos mismos.

Al finalizar el siglo XIV nos vamos a encontrar, en Guipúzcoa, con una pléyade de núcleos urbanos de modestas dimensiones (varían estas desde las 6,4 Ha. de San Sebastián, hasta $1 \mathrm{Ha}$. de Elgueta), en los que hay que articular su espacio de acuerdo con el nuevo espíritu urbano, esto es, hay que repartirlo entre todos los posibles pobladores que quieran establecerse en ellos. Sabemos también que dentro del recinto intramuros el espacio parcelado para su utilización con fines privados, constituye la mayor parte del suelo urbano.

Todas las referencias sobre la forma de repartir los solares las encontramos en la documentación perteneciente al siglo XIV. En los primeros años de este siglo, en la Carta-Puebla de Azpeitia -1310-, ya se dice que los terrenos otorgados por el rey para edificar la villa, sean repartidos igualmente entre todos. Años más tarde en otra Carta Puebla, la de la vecina Miranda de Iraurgui (Azcoitia), se insiste en el tema. A finales del siglo XIV, y en la fundación de la última villa del período medieval, Villarreal de Urrechua, las disposiciones para la partición de los solares son muy precisas. Al igual que en el caso de Azpeitia se buscan "omes buenos" que hagan la partición de los solares.

En la villa de Azpeitia los solares serán iguales para todos los vecinos, en cambio en Villarreal se establecen dos modelos, uno mayor y 
otro menor. El solar propiamente dicho, o el más característico era el de mayores dimensiones, el de seis brazadas en ancho por nueve de largo, puesto que el otro era simplemente un «medio solar». Otro dato importante que aporta la documentación es el de asegurarnos que el solar mayor, el de seis por nueve brazadas ( $8 \times 12$ metros), era el de uso general en toda Guipúzcoa. Por tanto las medidas que ofrece la Carta Puebla de Villarreal no son únicas o excepcionales para dicha villa, sino que se está haciendo referencia a unas medidas de carácter general para todos los solares intramuros que se repartieron en todas las villas guipuzcoanas.

La afirmación de la similitud de los solares en todo el territorio de Guipúzcoa no sólo viene dada por las referencias documentales, sino por los resultados de la aplicación del método arqueológico, que constata y confirma dicha hipótesis. Al emprender un trabajo de campo por toda la provincia, aún hoy día, se puede comprobar en aquellos lugares donde "el progreso" no ha destruido todos los vestigios de la antigüedad, que los solares de las viviendas mantienen unas medidas constantes. Ocho metros de fachada a la calle por doce metros de fondo. Estas medidas se corresponden estrictamente con la proporción que nos ofrecen los datos numéricos de ia Carta-Puebla de Villarreal de Urrechua.

Pero si esto sucede y se repite constantemente en lo que podríamos denominar casa común, más sorprendente es aún lo descubierto sobre las casas-torre y casas-palacios situadas intramuros. Al medir las fachadas de éstas constatamos que en la mayor parte de los casos ocupan exactamente dos unidades de solares. Concretamente la casa de Berriatúa en Motrico mide dieciséis metros de fachada (8 por 2), y la casa de Basozabal en Azpeitia otros dieciséis. También se da el caso de casaspalacios que ocupan las dimensiones correspondientes a solar y medio. Por lo que hemos podido constatar no se dan medidas arbitrarias, sino que siempre aparecen reflejadas las antiguas particiones.

Estas antiguas particiones se reflejan también en aquellos lugares donde tenemos noticias de que ha habido una gran densidad de población y que por dicha causa fue necesario dividir los solares. También en este caso hemos podido comprobar que esos solares fragmentados, mantienen una proporcionalidad respecto a las medidas originales. Las dimensiones de sus fachadas a la calle son de cuatro metros (8:2). Ejemplos de estas viviendas construidas en los espacios de medios solares pueden verse todavía hoy en algunas zonas de la villa de Fuenterrabía. 
El espacio que correspondía a cada unidad familiar, en los primeros años fundacionales, era similar para todos los vecinos, pero ya en el siglo XV esta situación, en algunas villas, varía notablemente. El solar guipuzcoano sigue manteniendo la misma medida, sin embargo el espacio ocupado por cada familia ya no se corresponde en todos los casos con esa unidad de espacio urbano edificable. En aquellas villas que resultaron atrayentes, debido a su prosperidad económica, el módulo de la vivienda familiar se reduce respecto a épocas anteriores. Al acudir más pobladores de los previstos resulta imposible que cada vecino o unidad familiar ocupe un solar completo. Las quejas más insistentes respecto a lo reducido de los solares se dan en la villa de San Sebastián. Al obligar el concejo a edificar las paredes medianeras de las casas en piedra, los vecinos oponen resistencia pues dichas paredes ocupan, según sus criterios, gran parte de la superficie habitable, prefieren seguir construyéndolas con tablas, ya que este sistema de separación ocupaba menos espacio.

El espacio urbano escaso en la mayor parte de las villas, se muestra insuficiente durante el siglo XV para algunas de las villas guipuzcoanas. Ya desde finales del siglo XIV se acaban de ocupar los espacios edificables en el interior de las cercas. Como resultado de ello a lo largo del siglo XV se fragmentan los solares, se crean y crecen los arrabales y se intentá, por parte de los vecinos, la ocupación paulatina de los espacios públicos intramuros.

Con la ocupación de dichos espacios no se logra aumentar de forma ostensible el número de solares, pues seria un hecho excesivamente llamativo para las autoridades concejiles. Sin embargo se trata de un fenómeno lento pero constante, además se vio favorecido por las continuas destrucciones de viviendas provocadas por los incendios. Al quedar destruidas las casas, éstas tienen que volver a reconstruirse en el menor espacio de tiempo posible, con lo que se favorece la falta de control, de esta manera el espacio público urbano, lentamente se va modificando o mejor dicho, se va reduciendo en perjuicio de todos y en beneficio de unos pocos.

Parece que en el último cuarto del siglo $x \vee$ y a partir de devastadores incendios que arrasaron totalmente algunas villas, los respectivos concejos se plantearon la reconstrucción de la población de una manera total y organizada. $Y$ es precisamente en este momento cuando se redactan Ordenanzas especificas sobre la construcción urbana (en San Sebastián 
y en Mondragón) ${ }^{2}$. En ellas se trataba de poner freno a la habitual costumbre de ocupación de los espacios públicos y también de incorporar nuevos materiales constructivos en las edificaciones de viviendas que fueran más resistentes a la acción destructora de los incendios.

Las primeras viviendas que los particulares construyeron dentro del recinto urbano tuvieron que someterse a las directricces que sobre edificación marcaron los primeros pobladores. Las primeras líneas de actuación sobre edificación de viviendas ya venían marcadas en las Cartas fundacionales. En ellas se ordenan repartir los solares con unas medidas determinadas y de forma similar para todos los vecinos, por tanto, desde el primer momento hay un control del suelo urbano, que configurará las posteriores construcciones. Se puede decir que las primeras licencias de obras estaban implícitas en estos repartos de solares.

Los materiales de construción empleados en la Arquitectura popular deben reunir, para la época a la que nos referimos, tres requisitos esenciales: que se encuentren próximos a los núcleos de población, que sean abundantes, y que su coste no resulte elevado para los vecinos.

El material básico en la construcción medieval guipuzcoana fue la madera. Prácticamente la totalidad del edificio se construía con dicho material. Sabemos también que hasta el último cuarto del siglo $\mathrm{xV}$ no se introdujo ningún otro material. Los guipuzcoanos no tienen ningún problema para hacerse con la madera necesaria para la construcción de sus viviendas, los vecinos de cada villa tenían acceso franco a los montes de sus términos jurisdiccionales, para cortar en ellos la madera que les fuera necesaria para la realización de sus casas. En 1345 en la Concordia sobre demarcación de límites y disfrute de montes, que se establece entre las villas de Deva, Elgoibar y el lugar de Mendaro, se decreta que los vecinos de estas tres localidades podrán utilizar libremente la madera de dichos montes para construir sus casas.

Las maderas más utilizadas en la construcción de edificios eran sin lugar a dudas, el roble y el haya, aunque también se utilizaron en menor medida el sauce, el fresno y la madera de aliso.

2 Rectificación de las Ordenanzas de Construcción de San Sebastián de 1491. Publicadas por C. ECHEGARAY CORTA, en Investigaciones históricas referentes a Guipúzcoa, Edit. La Gran Enciclopedia Vasca, t. X Bilbao, págs. 336-347.

Archivo General de Simancas. Registro General del Sello. 1490-V-Folio 379. 
Las villas adquirieron una fisonomía muy similar en todos los casos. Todas las partes de las viviendas estaban realizadas en dicho material. Empleaban vigas para la estructura y soporte de la vivienda, y tablas para el cierre exterior y también para su tabicación interior. En los primeros años también era de madera la cubierta, colocándose tablillas a modo de tejas. En 1485 los vecinos de Elgoibar, en un documento dirigido a los reyes, explican la situación de la villa y la de todos los guipuzcoanos alegando: "que la villa con sus arrables estan edificados en forma muy peligrosa, e cerca del peligro del fuego, porque así como comunmente eran en toda esa dicha provincia, las casas de madera, y estavan juntas e apegadas las unas a las otras, de guisa que todos estan en peligro de una sola candela que se pusiese a mal recabdo, por lo qual se solian quemar o quemaban muchas villas e logares desa dicha provincia» ${ }^{3}$.

Aunque en el siglo $x v$ todavía muchas de las construcciones fueran realizadas en madera, a partir del último cuarto de ese siglo, se alzan voces airadas, o por lo menos alarmadas, afirmando que dicha costumbre era totalmente perniciosa para la colectividad, ya que al más mínimo descuido, la villa entera podía quedar destruida.

Los concejos al tiempo que ordenan edificar las casas en piedra, se preocupan de que el material necesario para la reconstrucción de las viviendas esté dispuesto y a punto para los vecinos. Para ello se hacen llamamientos a todos aquellos que trabajan en la extracción de la madera o de la piedra, así como a los que tuvieran cal, yeso y otros materiales imprescindibles para la edificación, para que tuvieran abundante material dispuesto y para que mantuvieran los precios. Sabemos que a mediados del siglo XVI San Sebastián tenía construida la mayor parte de sus viviendas en cal y canto y argamasa, obedeciendo de esta forma las disposiciones concejiles de finales del siglo XV.

La edificación a base de iadrillo no fue muy corriente en Guipúzcoa, comenzándose a utilizar dicho material muy tardiamente. Han aparecido vestigios de ladrillos en excavaciones realizadas en el País Vasco referidas al siglo XIV. Sin embargo uno de los primeros momentos en que se utiliza el ladrillo de forma generalizada, es en la reconstrucción de las

3 A. Municipal de Deva. Libro V. n. ${ }^{\circ} 17$ Folio 89. AGS-RGS. 1485-V-Folio 145. 
partes superiores de las torres desmochadas de los Parientes Mayores. Pero fundamentalmente desde finales del siglo $x \mathrm{v}$, coincidiendo con la obligatoriedad de construir con otros materiales que no fuera la madera.

También se introduce un «nuevo» sistema constructivo que había estado ausente en la Guipúzcoa medieval, pero que había sido utilizado en otras zonas peninsulares, me refiero al «verganazo». Todavía se pueden encontrar ejemplos de este sistema constructivo en algunas zonas rurales. Se empleaba fundamentalmente en interiores. Se trataba de fabricar paredes con ramas de setos trenzados, generalmente ramas de avellanos, que recubiertos de barro hacían las veces de tabiques de partición interior. El concejo de Mondragón lo plantea como una de las soluciones, al igual que el empleo de la piedra, tapia o ladrillo, pera evitar los incendios ${ }^{4}$.

El cambio de fisonomía en las viviendas guipuzcoanas comenzará a producirse a partir de los últimos veinte años del cuatrocientos. Hay una ruptura brusca en la arquitectura popular urbana, se imponen nuevos materiales y nuevas condiciones, influidos por el temor a los incendios. Este cambio va a afectar fundamentalmente al tipo de materiales empleados, pero también afectará, aunque de forma más débil, a las formas arquitectónicas del edificio, ya que en muchas de las Ordenanzas se regula la altura de las casas, las dimensiones de los saledizos, y la anchura de las paredes medianeras.

Podemos decir que la arquitectura popular guipuzcoana se verá alterada por la legislación que los mismos vecinos creen conveniente aplicar, para evitar males mayores. En este caso no se puede hablar de un proceso paulatino de transformación y modificación de la arquitectura popular, sino de un salto brusco. Para conseguir este cambio se "privilegian" las casas de piedra sobre las de madera, dándoles prioridad a las fabricadas de cal y canto, pudiendo éstas elevarse por encima de las de madera, e impidiendo que las de madera perjudiquen o estorben a las de piedra.

Aunque en la mente de todos los vecinos estaba clara la necesidad de cambiar los materiales constructivos, parece que la inercia y la tradición pesaron más que la racionalidad, puesto que como se aprecia a

4 Ordenanzas de Edificación de Mondragón Capítulo III.

AGS. Cámara de Castilla. Pueblos Leg. 13. 
través de la documentación, la resistencia popular al cambio fue más intensa de lo que cabría esperar. Las Ordenanzas de Construcción de la villa de San Sebastián se redactaron en 1489, pero dos años más tarde se realizan unas rectificaciones a dichas Ordenanzas, en las que se amplía considerablemente el plazo para la edificación según las nuevas normativas. El primer plazo comprendía de 1489 a 1490 , parece que fue imposible reconstruir las viviendas con los nuevos materiales en tan breve espacio de tiempo, y en 1491 se amplía éste hasta 1496. En la misma Ordenanza se estimula a los vecinos a construir con ladrillo o cal y canto, asegurándoles que el coste de la casas hechas en madera y tabla era muy similar al de las hechas en piedra o argamasa. Finalizado el segundo plazo, los vecinos solicitan a finales de 1496 una prórroga, alegando las cuantiosas pérdidas sufridas, y los Reyes otorgan nuevamente un tercer plazo de tres años para acabar de reconstruir la villa según el nuevo sistema constructivo.

Estas normativas estaban vigentes en casi todas las villas que hubieran sufrido destrucciones masivas. Mondragón redactó sus Ordenanzas de Construcción en 1489, que fueron aprobadas en 1490. En 1499, el 18 de marzo, los Reyes Católicos expidieron en Madrid una Real Provisión, por la que ordenaban que todas las casas de Tolosa se construyeran a partir de ese momento en cal y canto, y que fueran sustituidas las de madera por las de piedra, en un plazo de quince años. En 1514 se decreta que ni en Deva ni en sus arrabales se pueden construir casas de madera y tablas ${ }^{5}$.

Las viviendas medievales de los núcleos urbanos guipuzcoanos no se planteaban como unidades autónomas dentro del conjunto urbano, sino que constituían un todo homogéneo, donde la separación entre unas y otras, era una división puramente ocasional en el conjunto serializado de las parcelas medievales. Aun dentro de esta homogeneidad, las únicas que escapaban de la tota! uniformidad eran las casas fuertes, casas torres y los palacios. Las casas torres debido en parte a la configuración de la parcela, y a la ejecución del edificio. En cambio, las casas palacio, que también destacaban sobre el conjunto urbano, lo hacían, no tanto, por la diferenciación parcelaria, como por la elegancia del diseño y los materiales empleados.

\footnotetext{
${ }^{5}$ A. Municipal de Deva. Libro VII Doc. $n .{ }^{\circ} 2$ Folio 9.
} 
A través de lo expuesto hasta el momento, se pueden apreciar, ya, algunos rasgos de lo que fue la vivienda de la inmensa mayoría de los vecinos de las villas. Se ha hablado de los solares, y ya hemos indicado que la tendencia general en Guipúzcoa era la de una distribución homogénea de los suelos edificables dentro de los recintos urbanos, por tanto en este punto la mayoría de las viviendas presentaban un aspecto similar. $Y$ no hay que olvidar que dichos lotes de tierra, estaban formando parte de una estructura urbanística más amplia, formado por el seriado gótico, que producirá manzanas de casas compactas que darán origen a las calles. El otro aspecto que unifica el conjunto de las viviendas urbanas, es el material constructivo empleado.

Con un espacio similar para cada familia y con una técnicas y materiales semejantes, el producto resultante necesariamente tiene que ser homogéneo. Además hay que recordar que también la modificación que sufren las viviendas en el último cuarto del siglo $\mathrm{xV}$, fue general en el ámbito de la Provincia. Primero unas villas y a continuación las otras, van ordenando a sus vecinos sustituir la madera por la piedra o cal y canto, con lo cual toda la fisonomía de la vivienda urbana irá modificándose a la vez. Por tanto la vivienda urbana común es muy semejante a lo largo de toda la Edad Media para todos los moradores de las villas.

Al igual que en la mayor parte de los núcleos urbanos de la época, la parcela correspondiente a cada vecino era estrecha y profunda. La casa urbana prima en importancia la fachada que da a la calle, ello se debía fundamentaimente al tipo de actividad económica realizada de carácter artesanal o mercantil, cuyo valor material residía no sólo en la superficie de su terreno a edificar, sino también en la mayor o menor posibilidad de sacar huecos a la calle. Se trataba de conseguir por to menos dos huecos a la calle, el de acceso a la vivienda y el de la tienda o almacén. Se configurarán por tanto las parcelas como estrechas y profundas en la proporcioón de uno a uno y medio.

A partir de la segunda mitad del siglo $x \mathrm{~V}$, los concejos empiezan a ordenar a sus moradores a que construyan sus casas en piedra, y es entonces cuando surge el grave problema de la pared medianera. Es bien sabido por todos que la edificación en piedra dificultaba la propagación del fuego de unos solares a otros, sobre todo en el caso de la situación de los solares guipuzcoanos que eran todos contiguos y estaban alineados formando una calle. Pero esta medida, beneficiosa en sí para la comunidad, encontraba dificultades para ser llevada a la práctica. El motivo que alegaban para el incumplimiento de dicha ordenanza, era 
la pequeñez de sus solares y la reducción que iban a sufrir por este motivo en su espacio útil.

Las paredes medianeras alcanzaban un grosor de 55 a $60 \mathrm{~cm}$, que son medidas respetables para la proporción de los solares a los que nos estamos refiriendo. Un solar que se cerrara con paredes medianeras de tabla, tendría una superficie útil de aproximadamente 96 metros cuadrados, frente a los 88,8 metros cuadrados, que dispondría si cerrara su solar como indicaban sus ordenanzas. Pero esta reducción del espacio habitable se hacía todavía más notorio en los medios solares. Un medio solar edificado todo él en tabla, tendría una superficie útil de 48 metros cuadrados, pero si los dueños de ese mismo solar construyeran sus medianeras en piedra, sólo dispondrian de 40 metros cuadrados.

Respecto a la utilización del solar adjudicado a cada unidad familiar, hay que distinguir dos partes: lo edificado y el espacio abierto, este espacio abierto solía dedicarse a huerta, corral o patio. En la primitiva instalación de la población no se ocupó todo el solar con la edificación de la vivienda, ya que se destinaba el fondo de dicho solar a actividades propias del mundo rural. Se edificaban los dos tercios del solar, quedando por tanto una planta de vivienda totalmente cuadrada de 8 por 8 , reservándose los 32 metros cuadrados restantes del fondo del solar, para el cultivo de algunas hortalizas, o como patio en el que se recluían a los animales domésticos.

Pero en la mayor parte de los casos, este espacio abierto fue ocupado desde muy pronto por la ampliación de la vivienda. Primeramente los solares se fragmentan longitudinalmente, dando origen a dos unidades de viviendas familiares, que al ser tan reducidas, ocupan inmediatamente los fondos de los solares. $Y$ es así como nos encontramos con lo que algunos arquitectos liaman «solar gótico», muy estrecho y alargado, que en nuestro caso sólo se llega a él como producto final de una evoIución del solar medieval que tiene su orígen en los siglos XIII y XIV.

Al hablar de la superficie edificada no podemos olvidar el tema de los «voladizos». Las estructuras de madera permitían la fácil construcción de voladizos en las plantas superiores. Cada piso avanzaba sobre la calle con respecto al anterior, y se llegaba en algunas calles no muy anchas, a estar tan juntas las casas, que sus tejados prácticamente se tocaban. Contra esta situación y excesos regulan las Ordenanzas de Construcción de San Sebastián y Mondragón. En la villa de Mondragón se prohibe a los propietarios de las viviendas avanzar en cada sobrado 
más de medio codo sobre el lienzo de la pared inferior, y esto en dos sobrados únicamente. San Sebastián permitía a sus vecinos en sus Ordenanzas de Construcción de 1489, que sacaran corredores a la calle en todas las casas construidas en cal y canto. Es generoso este Concejo en la cesión del espacio público, pues frente al codo que se permitía en Mondragón en cada uno de los sobrados, éste permitía hasta tres codos desde el primer sobrado, no pudiendo sobresalir más que esta medida en los pisos superiores.

La vivienda urbana durante sus primeros años de vida se caracterizó por su escaso desarrollo en altura, las primitivas casas eran de dos alturas, planta baja y sobrado. Sin embargo pronto comenzaron a desarrollarse en altura. A pesar de todo el control ejercido por los concejos, la tendencia a construir en altura es imparable, ya que no todas las villas disponían de espacios libres para expansionarse. Esta tendencia se ve favorecida por las nuevas normativas en materia de construcción, ya que al obligar a los vecinos a construir sus casas en cal y canto, y a realizar unas paredes medianeras en piedra, los vecinos deciden amortizar el gasto, edificando mayor número de pisos en cada solar. Parece ser que en los primeros años del siglo XVI algunas de las villas consiguen legalizar el tercer sobrado, así el 26 de abril de 1510, la Reina doña Juana libró una provisión a la villa de Tolosa por la que se les permitía elevar sus viviendas hasta los veinticuatro codos ${ }^{6}$.

Poco podemos decir respecto a la distribución interna de las viviendas. La planta baja cumple una función fundamental y similar en todas las viviendas. Además de ser el paso obligado para llegar a los sobrados y a los huertos o patios posteriores, cumplía otras funciones. Era el lugar donde los artesanos trabajaban en sus oficios, o donde los comerciantes despachaban sus productos a través de sus tableros. En esta planta baja se situaban asimismo pequeñas cuadras o corrales para los animales domésticos. Los huecos a la calle de esta planta baja solían ser dos, la puerta de acceso, muy amplia, para dar paso a personas y animales y para proporcionar luz y ventilación. El otro hueco era la ventana que además de proporcionar luz al interior, servía como mostrador o lugar de despacho de mercancías.

6 P. Gorosabel: Diccionario...

La Gran Enciclopedia Vasca. Bilbao, t. IV 1971, págs. 509-510. 
La residencia familiar o el lugar de reposo estaba situado en el primer sobrado. Los huecos no eran numerosos, pero sí relativamente amplios. A la fachada daban dos habitaciones, siempre que el solar no estuviera fragmentado. El segundo sobrado podía ser utilizado como lugar de almacenamiento de productos tales como pajas, linos, frutas, o cualquier otro material necesario para el quehacer habitual de una familia. Si el solar estuviera ocupado de forma plurifamiliar, en este segundo sobrado se repetiría el esquema del primer sobrado. En caso de haber un tercer sobrado el esquema se volveria a repetir.

Los vecinos en su intento continuado de ganar espacio útil en el interior de las viviendas, no dudan en "ampliar» sus casas a costa del espacio público. Una de las prácticas más habituales consistía en desarrollar la actividad profesional de cada uno fuera de sus propios locales, como el caso de los carniceros que utilizaban la vía pública para despiezar y colgar los animales sacrificados. $O$ de cualquier otro artesano que por medio del sistema de "tableros" más o menos amplios, extendía su mercancia en el espacio público. De esta forma el espacio privado quedaba temporalmente desembarazado, permitiendo una mayor comodidad en su interior. Pero si bien esta forma citada de ocupar los espacios públicos, lo hacía de forma temporal, esto es durante el día, existía otra forma de ocupación del espacio público de forma continuada, que favorecía iambién la ampliación del espacio útil de las viviendas urbanas, me refiero a las escaleras de piedra, exteriores a la vivienda, que daban acceso a los distintos sobrados de la casa.

Los núcleos urbanos guipuzcoanos debido a su compactación y a su sistema constructivo a lo largo de la Edad Media resultaron extremadamente vulnerables. Al ser la madera el material constructivo por excelencia, el riesgo de las villas ante los incendios fue inmenso. Además, estas posibilidades de propagación del fuego en los recintos urbanos aumenta con los materiales altamente combustibles que se guardaban dentro de las villas como grasa, sebo, alquitrán, pajas, linos, etc.

La mayor parte de los núcleos guipuzcoanos así como los de otras zonas próximas, se vieron consumidos innumerables veces, total o parcialmente, por el fuego. El número de incendios que soportaron las villas fue realmente alto.

San Sebastián se quema en nueve ocasiones. 
Mondragón es destruida por el fuego en cuatro ocasiones.

Segura se incendió en tres ocasiones, al igual que Tolosa.

Rentería, Fuenterrabía y Azpeitia padecieron dos incendios cada una.

Azcoitia, Salinas de Léniz, Villafranca, Hernani y Motrico, fueron reducidas a cenizas por lo menos una vez.

De los trece núcleos restantes carecemos de noticias respecto a los incendios que pudieron haber sufrido, sin embargo, la carencia de noticias documentales directas, no nos permite afirmar que dichos núcleos no hubieran sufrido semejante desgracia.

A pesar de las continuas destrucciones que sufrieron las villas, éstas no fueron abandonadas más que por el breve espacio de tiempo que tardaban los vecinos en reconstruir sus viviendas. Este sistema de poblamiento y de vida fue aceptado plenamente por la población guipuzcoana, que a pesar de su vulnerabilidad y de las frecuentes destrucciones sufridas, se empeñan en mantenerlo, reconstruyendo continuamente el medio físico que había dado origen a una nueva forma de vida, la urbana. 


\section{EVOLUCION DE LAS FACHADAS}

12 CODOS

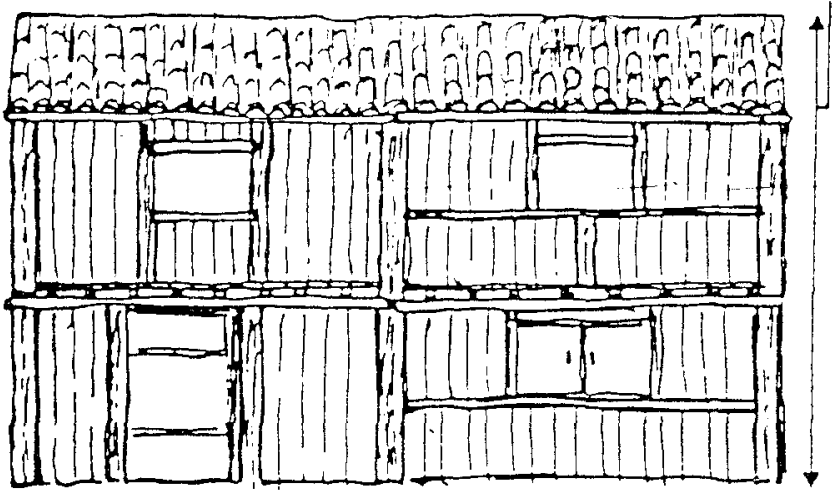

S. $x\|11 \cdot x\| y^{1}$

$19 \operatorname{coDOS}$

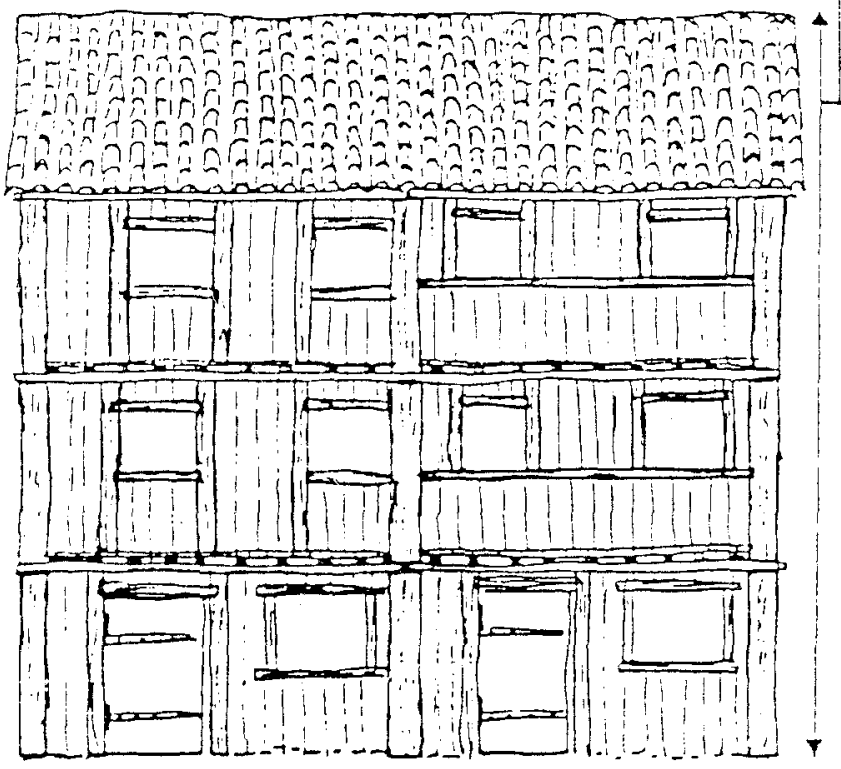

S.XIY $Y^{2}-X V$ 


\section{EVOLUCION DE LAS FACHADAS}

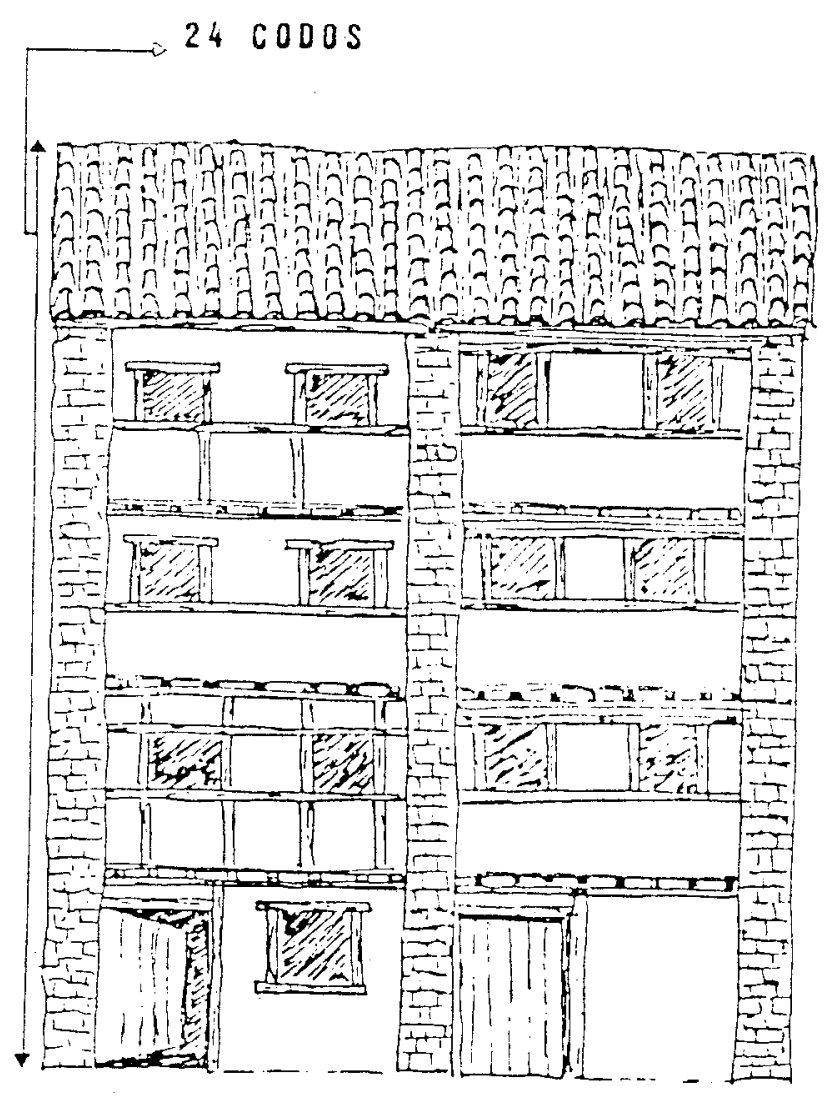

\section{$S \cdot X Y 1$}


BEATRIZ ARIZAGA BOLUMBURU

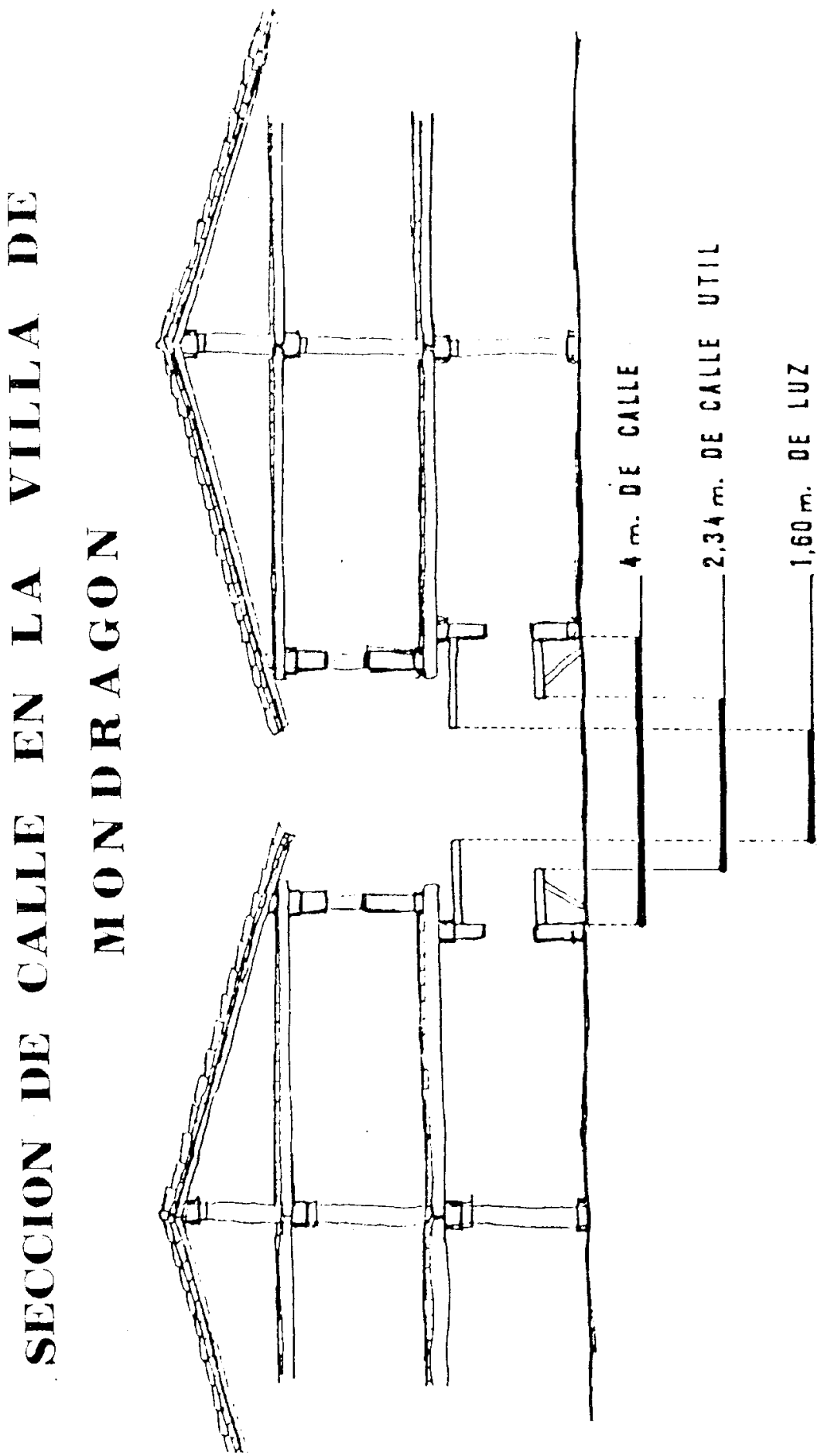

